Hans Petter Langtangen

\title{
A Primer an Scientific Programming with Python
}

421 Springer 


\section{Contents}

1 Computing with Formulas

1.1 The First Programming Encounter: A Formula ............. 1

1.1.1 Using a Program as a Calculator ........................... 2

1.1.2 About Programs and Programming ..................... 2

1.1.3 Tools for Writing Programs .................................... 3

1.1.4 Using Idle to Write the Program ……………........ 4

1.1.5 How to Run the Program ......................................... 7

1.1.6 Verifying the Result .................................................. 8

1.1 .7 Using Variables .......................................................... 8

1.1.8 Names of Variables ..................................................... 9

1.1.9 Reserved Words in Python ...................................... 10

1.1 .10 Comments ................................................................. 10

1.1.11 Formatting Text and Numbers ............................. 11

1.2 Computer Science Glossary .................................................... 13

1.3 Another Formula: Celsius-Fahrenheit Conversion .......... 18

1.3.1 Potential Error: Integer Division .......................... 19

1.3.2 Objects in Python ………………………….......... 20

1.3.3 Avoiding Integer Division ...................................... 21

1.3.4 Arithmetic Operators and Precedence ................ 21

1.4 Evaluating Standard Mathematical Functions ................. 22

1.4.1 Example: Using the Square Root Function ........ 22

1.4.2 Example: Using More Mathematical Functions 25

1.4.3 A First Glimpse of Round-Off Errors .................. 25

1.5 Interactive Computing ………………………………….... 26

1.5.1 Calculating with Formulas in the Interactive Shell ................................................................... 27

1.5.2 Type Conversion .................................................... 28

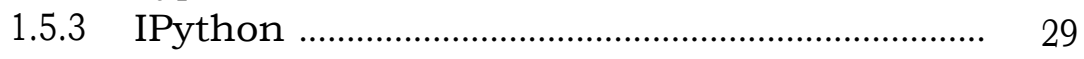

1.6 Complex Numbers ......................................................... 31 
1.6.1 Complex Arithmetics in Python ........................... 32

1.6.2 Complex Functions in Python ................................. 32

1.6.3 Unified Treatment of Complex and Real Functions …………………………………........ 33

1.7 Summary .......................................................................... 35

1.7.1 Chapter Topics ........................................................ 35

1.7.2 Summarizing Example: Trajectory of a Ball ... 38

1.7.3 About Typesetting Conventions in This Book . 39

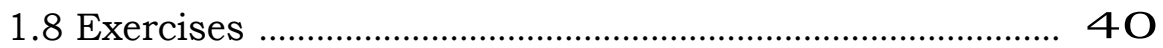

2 Basic Constructions ......................................................... 51

2.1 Loops and Lists for Tabular Data ................................... 51

2.1.1 A Naive Solution ................................................ 51

2.1.2 While Loops ………………………………........ 52

2.1.3 Boolean Expressions …………………………... 54

2.1.4 Lists …. 56

2.1.5 For Loops ……………………………………...... 58

2.1.6 Alternative Implementations with Lists and

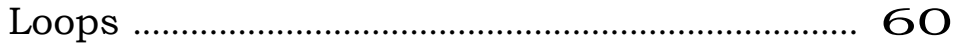

2.1.7 Nested Lists ............................................................. 64

2.1.8 Printing Objects ..................................................... 65

2.1.9 Extracting Sublists .................................................... 66

2.1.10 Traversing Nested Lists ........................................... 68

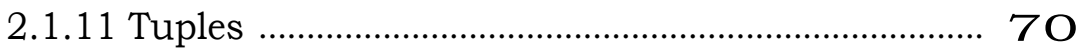

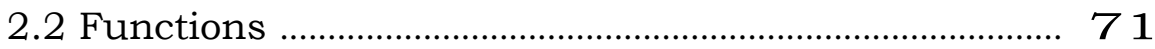

2.2.1 Functions of One Variable .................................... 71

2.2.2 Local and Global Variables .................................... 73

2.2.3 Multiple Arguments .................................................. 75

2.2.4 Multiple Return Values ........................................ 77

2.2.5 Functions with No Return Values ........................ 79

2.2.6 Keyword Arguments .............................................. 80

2.2.7 Doc Strings .............................................................. 83

2.2.8 Function Input and Output .................................. 84

2.2.9 Functions as Arguments to Functions ............... 84

2.2.10 The Main Program …………………………........ 86

2.2.11 Lambda Functions ……………………………..... 87

2.3 If Tests ....................................................................... 88

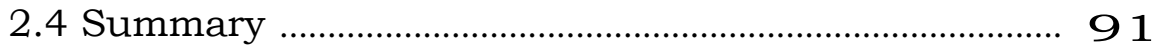

2.4.1 Chapter Topics ...................................................... 91

2.4.2 Summarizing Example: Tabulate a Function. $\quad 94$

2.4.3 How to Find More Python Information .............. 98

2.5 Exercises …………………………………………......... 99

3 Input Data and Error Handling …………………...... 119

3.1 Asking Questions and R,eading Answers ........................... 120 
3.1.1 Reading Keyboard Input ..................................... 120

3.1.2 The Magic "eval" Function ..................................... 121

3.1.3 The Magic "exec" Function ..................................... 125

3.1.4 Turning String Expressions into Functions ........ 126

3.2 Reading from the Command Line .................................... 127

3.2.1 Providing Input on the Command Line .............. 127

3.2.2 A Variable Number of Command-Line Arguments …………............................................. 128

3.2.3 More on Command-Line Arguments .................... 129

3.2.4 Option-Value Pairs on the Command Line ........ 130

3.3 Handling Errors ................................................................. 132

3.3.1 Exception Handling .............................................. 133

3.3.2 Raising Exceptions …………………………........ 136

3.4 A Glimpse of Graphical User Interfaces ......................... 139

3.5 Making Modules ……………………………………....... 141

3.5.1 Example: Compund Interest Formulas ................. 142

3.5.2 Collecting Functions in a Module File ................. 143

3.5.3 Using Modules ...................................................... 148

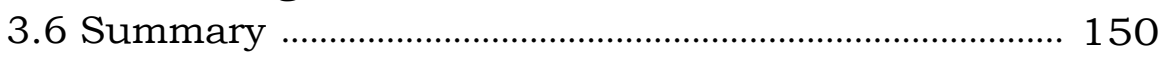

3.6.1 Chapter Topics ....................................................... 150

3.6.2 Summarizing Example: Bisection Root Finding 152

3.7 Exercises ......................................................................... 160

4 Array Computing and Curve Plotting ……............. 169

4.1 Vectors ............................................................................ 170

4.1.1 The Vector Concept …………………………....... 170

4.1.2 Mathematical Operations on Vectors ................... 171

4.1.3 Vector Arithmetics and Vector Functions ......... 173

4.2 Arrays in Python Programs ............................................... 175

4.2.1 Using Lists for Collecting Function Data ............. 175

4.2.2 Basics of Numerical Python Arrays .................... 176

4.2.3 Computing Coordinates and Function Values .. 177

4.2.4 Vectorization .......................................................... 178

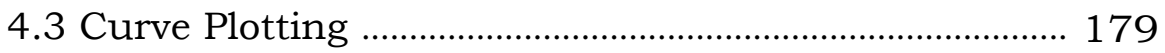

4.3.1 The SciTools and Easyviz Packages .................... 180

4.3.2 Plotting a Single Curve ........................................... 181

4.3.3 Decorating the Plot ............................................... 183

4.3.4 Plotting Multiple Curves ...................................... 183

4.3.5 Controlling Line Styles ........................................... 185

4.3.6 Interactive Plotting Sessions ................................ 189

4.3.7 Making Animations …………………………...... 190

4.3.8 Advanced Easyviz Topics ..................................... 193

4.3.9 Curves in Pure Text ............................................ 198

4.4 Plotting Difficulties ........................................................... 199

4.4.1 Piecewisely Defined Functions ............................... 199 
4.4.2 Rapidly Varying Functions ................................. 205

4.4.3 Vectorizing StringFunction Objects ................. 206

4.5 More on Numerical Python Arrays ................................ 207

4.5.1 Copying Arrays .................................................... 207

4.5.2 In-Place Arithmetics .......................................... 207

4.5.3 Allocating Arrays ............................................... 208

4.5.4 Generalized Indexing ......................................... 209

4.5.5 Testing for the Array Type ............................... 210

4.5.6 Equally Spaced Numbers ................................... 211

4.5.7 Compact Syntax for Array Generation ............... 212

4.5.8 Shape Manipulation ............................................ 212

4.6 Higher-Dimensional Arrays ........................................... 213

4.6.1 Matrices and Arrays .......................................... 213

4.6.2 Two-Dimensional Numerical Python Arrays 214

4.6.3 Array Computing ...................................... 216

4.6.4 Two-Dimensional Arrays and Functions of Two

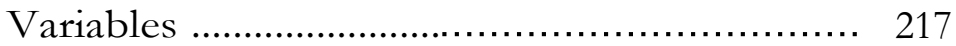

4.6.5 Matrix Objects ................................................ 217

4.7 Summary ……............................................................ 219

4.7.1 Chapter Topics ................................................. 219

4.7.2 Summarizing Example: Animating a Function . 220

4.8 Exercises ................................................................... 225

5 Sequences and Difference Equations .......................... 235

5.1 Mathematical Models Based on Difference Equations .. 236

5.1.1 Interest Rates ................................................... 237

5.1.2 The Factorial as a Difference Equation ............. 239

5.1.3 Fibonacci Numbers .......................................... 240

5.1.4 Growth of a Population ........................................ 241

5.1.5 Logistic Growth ............................................... 242

5.1.6 Payback of a Loan ............................................ 244

5.1.7 Taylor Series as a Difference Equation ................ 245

5.1.8 Making a Living from a Fortune ........................ 246

5.1.9 Newton's Method ............................................. 247

5.1.10 The Inverse of a Function ................................... 251

5.2 Programming with Sound ………………………........ 253

5.2.1 Writing Sound to File ……………………......... 253

5.2.2 Reading Sound from File .................................... 254

5.2.3 Playing Many Notes ........................................... 255

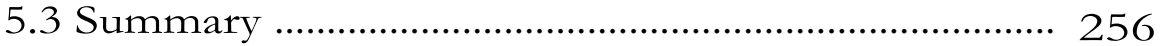

5.3.1 Chapter Topics ................................................ 256

5.3.2 Summarizing Example: Music of a Sequence $\quad 257$

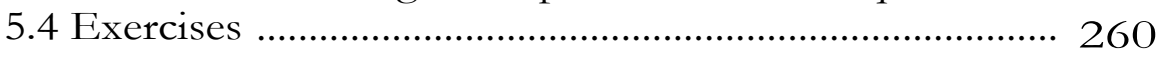

6 Files, Strings, and Dictionaries ...................................... 269 
6.1 Reading Data from File

269

6.1.1 Reading a File Line by Line

6.1.2 Reading a Mixture of Text and Numbers 273

6.1.3 What Is a File, Really? 274

6.2 Dictionaries 278

6.2.1 Making Dictionaries 278

6.2.2 Dictionary Operations 279

6.2.3 Example: Polynomials as Dictionaries 280

6.2.4 Example: File Data in Dictionaries 282

6.2.5 Example: File Data in Nested Dictionaries ........ 283

6.2.6 Example: Comparing Stock Prices ........................ 287

6.3 Strings 291

6.3.1 Common Operations on Strings ............................... 292

6.3.2 Example: Reading Pairs of Numbers ..................... 295

6.3.3 Example: Reading Coordinates ............................... 298

6.4 Reading Data from Web Pages ............................................ 300

6.4.1 About Web Pages ........................................................ 300

6.4.2 How to Access Web Pages in Programs ............... 302

6.4.3 Example: Reading Pure Text Files ....................... 302

6.4.4 Example: Extracting Data from an HTML Page 304

6.5 Writing Data to File .................................................................. 308

6.5.1 Example: Writing a Table to File ........................... 309

6.5.2 Standard Input and Output as File Objects .. $\quad 310$

6.5.3 Reading and Writing Spreadsheet Files ............... 312

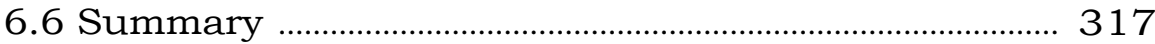

6.6.1 Chapter Topics ............................................................. 317

6.6.2 Summarizing Example: A File Database .............. 319

6.7 Exercises ….......................................................................... 323

7 Introduction to Classes .......................................................... 337

7.1 Simple Function Classes ....................................................... 338

7.1.1 Problem: Functions with Parameters .................... 338

7.1.2 Representing a Function as a Class ......................... 340

7.1.3 Another Function Class Example .......................... 346

7.1.4 Alternative Function Class Implementations . $\quad 347$

7.1.5 Making Classes Without the Class Construct . 349

7.2 More Examples on Classes …………................................... 352

7.2.1 Bank Accounts ....................................................... 352

7.2.2 Phone Book .................................................................. 354

7.2.3 A Circle ................................................................... 355

7.3 Special Methods ................................................................. 356

7.3.1 The Call Special Method .......................................... 357

7.3.2 Example: Automagic Differentiation ...................... 357

7.3.3 Example: Automagic Integration ............................ 360

7.3.4 Turning an Instance into a String ........................... 362 
7.3.5 Example: Phone Book with Special Methods ... 363

7.3.6 Adding Objects ........................................................ 365

7.3.7 Example: Class for Polynomials .............................. 365

7.3.8 Arithmetic Operations and Other Special Methods 369

7.3.9 More an Special Methods for String Conversion 370

7.4 Example: Solution of Differential Equations …………... 372

7.4.1 A Function for Solving ODEs ............................... 373

7.4.2 A Class for Solving ODEs ..................................... 374

7.4.3 Verifying the Implementation ………………........ 376

7.4.4 Example: Logistic Growth .................................... 377

7.5 Example: Class for Vectors in the Plane .......................... 378

7.5.1 Some Mathematical Operations an Vectors ........ 378

7.5.2 Implementation ....................................................... 378

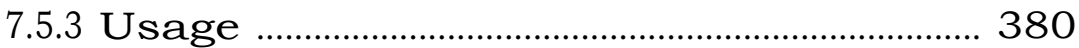

7.6 Example: Class for Complex Numbers ………………….. 382

7.6.1 Implementation .................................................... 382

7.6.2 Illegal Operations ..................................................... 383

7.6.3 Mixing Complex and Real Numbers .................... 384

7.6.4 Special Methods for "Right" Operands ................ 387

7.6.5 Inspecting Instances .................................................. 388

7.7 Static Methods and Attributes ……………………......... 389

7.8 Summary ……................................................................ 391

7.8.1 Chapter Topics ........................................................ 391

7.8.2 Summarizing Example: Interval Arithmetics . $\quad 392$

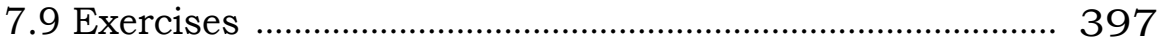

8 Random Numbers and Simple Garnes ...................... 417

8.1 Drawing Random Numbers ............................................... 418

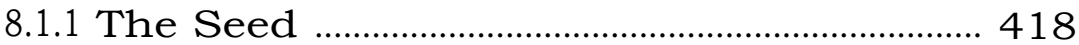

8.1.2 Uniformly Distributed Random Numbers .......... 419

8.1.3 Visualizing the Distribution .................................... 420

8.1.4 Vectorized Drawing of Random Numbers .......... 421

8.1.5 Computing the Mean and Standard Deviation .. 422

8.1.6 The Gaussian or Normal Distribution ................ 423

8.2 Drawing Integers ................................................................ 424

8.2.1 Random Integer Functions .................................... 425

8.2.2 Example: Throwing a Die ...................................... 426

8.2.3 Drawing a Random Element from a List ............ 427

8.2.4 Example: Drawing Cards from a Deck ................. 427

8.2.5 Example: Class Implementation of a Deck ....... 429

8.3 Computing Probabilities ....................................................... 432

8.3.1 Principles of Monte Carlo Simulation ................... 432

8.3.2 Example: Throwing Dice .......................................... 433

8.3.3 Example: Drawing Balls from a Hat .................... 435 
8.3.4 Example: Policies for Limiting Population

Growth

8.4 Simple Garnes

8.4.1 Guessing a Number

8.4.2 Rolling Two Dice 440

8.5 Monte Carlo Integration 443

8.5.1 Standard Monte Carlo Integration

8.5.2 Computing Areas by Throwing Random Points

8.6 Random Walk in One Space Dimension

8.6.1 Basic Implementation 448

8.6.2 Visualization 449

8.6.3 Random Walk as a Difference Equation 449

8.6.4 Computing Statistics of the Particle Positions .. 450

8.6.5 Vectorized Implementation 451

8.7 Random Walk in Two Space Dimensions .......................... 453

8.7.1 Basic Implementation 453

8.7.2 Vectorized Implementation ...................................... 455

8.8 Summary 456

8.8.1 Chapter Topics 456

8.8.2 Summarizing Example: Random Growth 457

8.9 Exercises 463

9 Object-Oriented Programming 479

9.1 Inheritance and Class Hierarchies 479

9.1.1 A Class for Straight Lines 480

9.1.2 A First Try an a Class for Parabolas 481

9.1.3 A Class for Parabolas Using Inheritance 481

9.1.4 Checking the Class Type 483

9.1.5 Attribute versus Inheritance 484

9.1.6 Extending versus Restricting Functionality ........ 485

9.1.7 Superclass for Defining an Interface 486

9.2 Class Hierarchy for Numerical Differentiation 488

9.2.1 Classes for Differentiation 488

9.2.2 A Flexible Main Program 491

9.2.3 Extensions 492

9.2.4 Alternative Implementation via Functions 495

9.2.5 Alternative Implementation via Functional Programming 496

9.2.6 Alternative Implementation via a Single Class

9.3 Class Hierarchy for Numerical Integration 499

9.3.1 Numerical Integration Methods 499

9.3.2 Classes for Integration 501

9.3.3 Using the Class Hierarchy 504

9.3.4 About Object-Oriented Programming 507 9.4 Class Hierarchy for Numerical Methods for ODEs 508 
9.4.1 Mathematical Problem ………………………………. 508

9.4.2 Numerical Methods …………………………………..... 510

9.4.3 The ODE Solver Class Hierarchy ……………….... 511

9.4.4 The Backward Euler Method …………………….... 515

9.4.5 Verification ........................................................................ 518

9.4.6 Application 1: $\mathrm{u}^{\prime}=\mathrm{u}$.................................................. 518

9.4.7 Application 2: The Logistic Equation ..................... 519

9.4.8 Application 3: An Oscillating System .................... 521

9.4.9 Application 4: The Trajectory of a Ball ............... 523

9.5 Class Hierarchy for Geometrie Shapes …………………..... 525

9.5.1 Using the Class Hierarchy …………………………... 526

9.5.2 Overall Design of the Class Hierarchy …………... 527

9.5.3 The Drawing Tool ……………………………………... 529

9.5.4 Implementation of Shape Classes ........................... 530

9.5.5 Scaling, Translating, and Rotating a Figure $\quad 534$

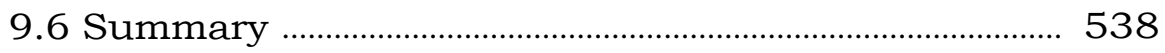

9.6.1 Chapter Topics ............................................................ 538

9.6.2 Summarizing Example: Input Data Reader ........ 540

9.7 Exercises ………………………………………………..... 546

A Discrete Calculus ......................................................... 573

A.1 Discrete Functions ………………………………………….... 573

A.1.1 The Sine Function …………………………………... 574

A.1.2 Interpolation ................................................................. 576

A.1.3 Evaluating the Approximation …………………….... 576

A.1.4 Generalization ………………………………….... 577

A.2 Differentiation Becomes Finite Differences ......................... 579

A.2.1 Differentiating the Sine Function …………………. 580

A.2.2 Differences an a Mesh ................................................ 580

A.2.3 Generalization ........................................................... 582

A.3 Integration Becomes Summation …………………………... 583

A.3.1 Dividing into Subintervals ...………………………. 584

A.3.2 Integration an Subintervals ………………………... 585

A.3.3 Adding the Subintervals ……………………………. 586

A.3.4 Generalization ……………………………………..... 587

A.4 Taylor Series ………………………………………………... 589

A.4.1 Approximating Functions Close to One Point . 589

A.4.2 Approximating the Exponential Function ........... 589

A.4.3 More Accurate Expansions ………………………..... 590

A.4.4 Accuracy of the Approximation ………………….... 592

A.4.5 Derivatives Revisited ............................................... 594

A.4.6 More Accurate Differente Approximations ........ 595

A.4.7 Second-Order Derivatives …………………………... 597

A.5 Exercises ……………………………………………………... 599 


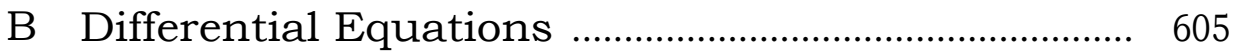

B.1 The Simplest Case ........................................................... 606

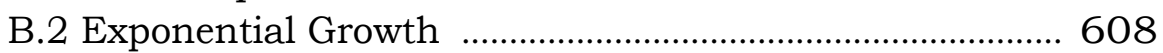

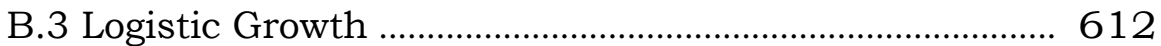

B.4 A General Ordinary Differential Equation ...................... 614

B.5 A Simple Pendulum ........................................................... 615

B.6 A Model for the Spread of a Disease …………………... 619

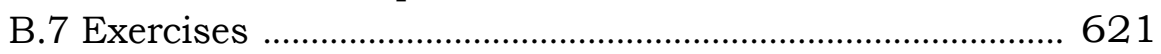

C A Complete Project ....................................................... 625

C.1 About the Problem: Motion and Forces in Physics ........ 626

C.1.1 The Physical Problem …………………………... 626

C.1.2 The Computational Algorithm ……………….... 628

C.1.3 Derivation of the Mathematical Model ………..... 628

C.1.4 Derivation of the Algorithm .................................... 631

C.2 Program Development and Testing ..................................... 632

C.2.1 Implementation ........................................................ 632

C.2.2 C allback Functionality .............................................. 635

C.2.3 Making a Module ……………………………....... 636

C.2.4 Verification …………………………………........ 637

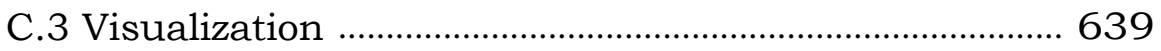

C.3.1 Simultaneous Computation and Plotting ............. 639

C.3.2 Some Applications ................................................... 642

C.3.3 Remark on Choosing Jät ....................................... 643

C.3.4 Comparing Several Quantities in Subplots ....... 644

C.3.5 Comparing Approximate and Exact Solutions $\quad 645$

C.3.6 Evolution of the Error as 3t Decreases .............. 646

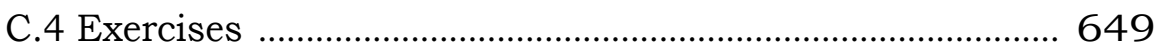

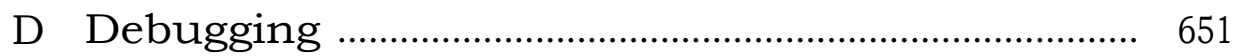

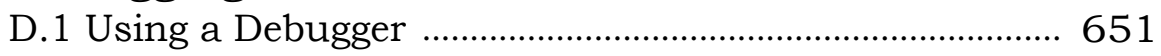

D.2 How to Debug ...................................................................... 653

D.2.1 A Recipe for Program Writing and Debugging .. 654

D.2.2 Application of the Recipe ........................................ 656

E Technical Topics ............................................................... 669

E.1 Different Ways of Running Python Programs ................ 669

E.1.1 Executing Python Programs in IPython ............. 669

E.1.2 Executing Python Programs on Unix .................. 669

E.1.3 Executing Python Programs on Windows .......... 671

E.1.4 Executing Python Programs on Macintosh ....... 673

E.1.5 Making a Complete Stand-Alone Executable .... 673

E.2 Integer and Float Division ................................................ 673

E.3 Visualizing a Program with Lumpy …………………….... 674

E.4 Doing Operating System Tasks in Python ……………... 675

E.5 Variable Number of Function Arguments ........................ 678 
E.5.1 Variable Number of Positional Arguments ........ 679

E.5.2 Variable Number of Keyword Arguments ........... 681

E.6 Evaluating Program Efficiency ……...................................... 683

E.6.1 Making Time Measurements ................................... 683

E.6.2 Profiling Python Programs ........................................ 685

Bibliography .......................................................... 687

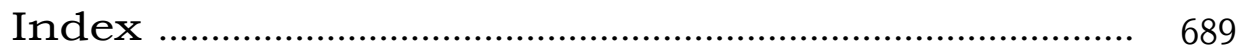

